\title{
Redirected walking for virtually expanded play area
}

\author{
Taiga Moriguchi ${ }^{1 *}$ and Michael Cohen ${ }^{1 * *}$ \\ ${ }^{1}$ Spatial Media Group, Computer Arts Lab; University of Aizu; Aizu-Wakamatsu, Fukushima 965-8580; Japan
}

\begin{abstract}
We describe a method of achieving redirected walking by modulating subjective translation and rotation. In a real space, a user walks around without leaving a $5 \mathrm{~m}^{2}$ area, but we have built a system that allows virtual movement around a larger area than the real space. This system is realized by translating and rotating the apparent ground in response to user movement.
\end{abstract}

\section{Introduction}

These days, with head-mounted displays (HMD) used in virtual reality (VR) becoming increasingly affordable, more people than ever can experience VR. Most VR content can be experienced using a user's direct body movements to induce immersion. However, the space to enjoy VR content (called "Guardian Space" by Oculus and "Chaperone" by SteamVR) can be limited. Idioms such as teleportation, where the user does not move in real space but moves from place to place in virtual space, are common in VR gameplay and experience. The use of actual movement improves one's sense of immersion in VR, so various methods have been developed to realize virtual space movement associated with actual movement.

One such method uses a locomotion interface such as a treadmill. A locomotion interface works to cancel out actual movement, providing a real walking sensation while keeping the user's location fixed [1]. Research is also being done on locomotion interfaces that can move up and down as well as on a flat surface [2]. This uses a motion platform that follows the positions of both feet and cancels out movement in each direction by having the other platform move in the exact opposite direction to the movement of each foot.

Virtuix's Omni [3] and Cybershoes' Cybershoes [4] products are available as locomotive interfaces for general VR content, allowing users to enjoy a walking VR experience. However, implementation of this method of walking is expensive and requires special equipment. Also, it restricts the user's activity because his or her body is fixed in place.

There is another method, called redirected walking (RDW), in which a user's virtual movements are manipulated to create different subjective imagery than the "real" one so that the user does not notice that he or she is moving in a virtual space different from the real one. Several methods have been studied to create this illusion, including one in which the user touches a wall curved in an arc to create the illusion by using haptic information as well as visual information [5], or another in which the user is shown imagery of the wind blowing from the front and the direction of the wind applied by a fan in the real space is gradually changed from the front. There is a method of guiding the user in an arbitrary direction by letting him or her walk on an unstable scaffold in a curved arc [6]. In addition to these visual and tactile methods, other methods have been proposed, such as dynamically placing sound sources along an intended footpath to guide the player [7], or showing a video of a very unstable scaffolding in a virtual space and having the player walk on the unstable scaffolding so that the player is acutely aware of the virtual risk and does not notice the difference between the two spaces [8].

These methods allow a user to walk more freely with less equipment than with a locomotion interface. However, to achieve this in a more general way, it is desirable to use as little equipment as possible. Therefore, we realize RDW by manipulating the amount of relative movement and rotation by moving and rotating the subjective ground in virtual space.

\section{Methodology}

\subsection{Redirected walking (RDW)}

RDW is a method of making a user feel as if they are moving in a vast world, even though they are moving in a limited physical space, by displaying imagery that is slightly different from the perspective of movement in real space.

Such motion can be divided into translation and rotation. In RDW, the difference between these two types of motion is created by scaling displayed value. In addition to these, there are also curvature operations that display scaled curved motion as linear motion [9].

In translational manipulation, actual movement can be virtually expanded or reduced by multiplying the

*morithin0512@gmail.com, ${ }^{* *}$ mcohen@u-aizu.ac.jp 
translation gain $g_{T} \in \mathbb{R}$ in the user's movement direction. Similarly, apparent rotation can be modulated by multiplying user's rotation by rotation gain $g_{\mathrm{A}} \in \mathbb{R}$.

The curvature operation converts motion on an arc into linear motion. Curvature gain is used for this conversion, defined by $g_{C}:=\frac{1}{r}$, where $\mathrm{r}$ is the radius of the circular arc.

In this study, we realize RDW using only translational and rotational operations to move and rotate the ground in response to user movement in real space. Curvature as such is unmodulated; its gain is 1 .

\subsection{Oculus Quest 2}

Oculus Quest 2 is a stand-alone VR HMD developed by Oculus [10]. While Oculus Go, also made by Oculus, is a 3-DoF (Degree of Freedom) HMD capable of continuously tracking orientation around the three axes of rotation (yaw, pitch, and roll), Quest is a 6-DoF, capable of detecting movement in the $\mathrm{x}, \mathrm{y}$, and $\mathrm{z}$ directions as well as rotations. The HMD is as good as any HMD that requires a PC, such as the company's Oculus Rift or HTC's VIVE, so users can enjoy highquality VR experience.

\subsection{Unity}

Unity is a game development engine with built-in IDE developed by Unity Technology [11]. Users can use C\# to write scripts for use in games and can develop games using prepared components and various functions. In recent years, it has been used not only for games but also for non-interactive content such as movies. The engine itself is compatible with various platforms, making it possible to build games for Windows, macOS, Linux, iOS, iPadOS, Android, the web, and set-top game consoles.

\subsection{Photon Unity Network (PUN)}

Photon Engine is a networking engine for distributed game development, developed by Exit Games [12]. When developing a distributed multiplayer game with client-server topology, it is necessary to prepare a server and specify URLs to access it. However, this engine and its service simplifies part of the process. PUN is a package of features necessary to use this framework for developing multiplayer games in Unity.

\section{Implementation}

\subsection{Overview}

\subsection{Realize RDW}

In this study, RDW was realized by using two parallel manipulations of the displacement (translation) and the rotation (azimuth). Gains used in this study are $g_{T}=2$ and $g_{\mathrm{A}}=\frac{2}{3}$. During development, we tried a value that would positively magnify the rotation $\left(g_{A}>1\right)$, but results wee unsatisfying. For example, a clockwise rotation of $270^{\circ}$ and a counterclockwise rotation of $90^{\circ}$ would result in the same final direction. Therefore, we thought it was unrealistic and did not use this

Oculus Quest 2 ( $1^{\text {st }}$-person View)

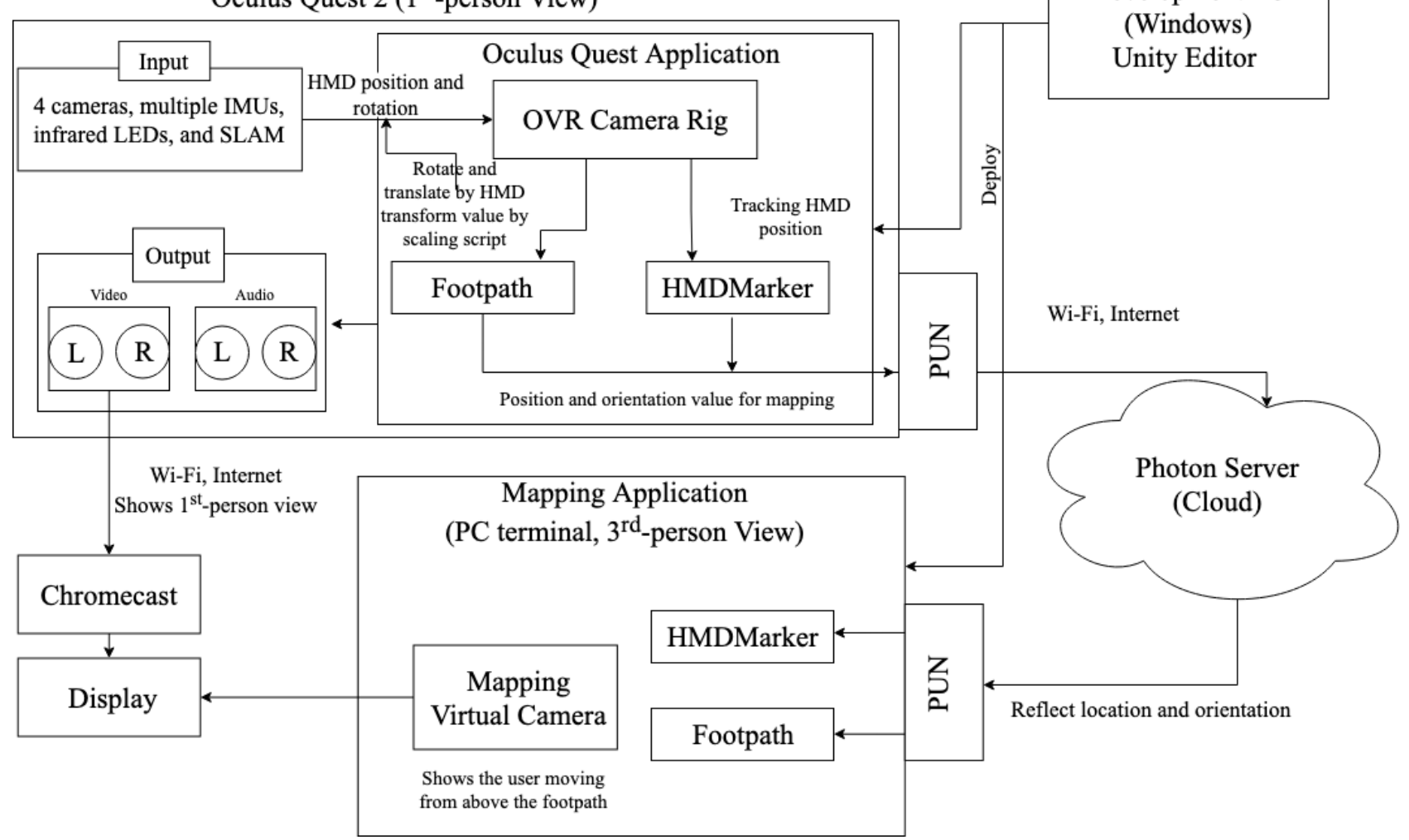

Fig. 1. System Architecture 
modulation. These gains were not directly multiplied by the HMD parameters, but the relative translational movement $T_{S}$ and azimuthal rotation $A_{S}$ of the scene in virtual space are manipulated based on the amount of translational movement $T_{O}$ and azimuthal rotation $A_{O}$ every frame of the HMD imagery. These parameters can be derived from the amount of translational movement $T_{G}$ and azimuthal rotation $A_{G}$ of the ground as follows.

$$
\begin{gathered}
T_{G}=T_{O}\left(1-g_{T}\right) \\
A_{G}=A_{O}\left(1-g_{\mathrm{A}}\right) \\
T_{S}=T_{O}-T_{G} \\
=T_{O} g_{T} \\
A_{S}=A_{O}-A_{G} \\
=A_{O} g_{A}
\end{gathered}
$$

\subsection{User Guidance}

Visualized effects were generated to improve the accuracy of guidance. In this study, we erected a path to walk along in the virtual space to keep users within the Guardian Space. Areas off the path were filled with (virtual) molten lava so users are psychologically discouraged from leaving the path. Spatial sound sources were placed at corners of the path, playing bell tones to aurally guide the user.

We created a path in the virtual space according to scaled translation and rotation described in the previous section after defining the path in real space, as shown in Fig. 2. Segment numbers shown in the subfigures correspond. The 3D model of the path was created by combining primitive elements included in Unity editor.
Oculus Quest creates an individual experience that cannot be properly viewed by anyone other than the person wearing the HMD, so we also developed a tracking and mapping function. To synchronize movement with the HMD, we used PUN to share location and orientation via Photon Cloud. A marker (HMD Marker) was placed above the user's icon to tarck the HMD's position and rotation, and the position of the marker was synchronized with PUN, allowing a user's movement along the path in the virtual space to be mapped.

\section{Result}

This software we developed allows a player to move through a larger virtual area than that available in real area by providing a VR play area of considerable size. The actual first-person view and mapping views are shown in Figs. 3 and 4. Since the longest straight line in the virtual space is $5 \mathrm{~m}$, if the prepared Guardian Space is larger than $25 \mathrm{~m}^{2}$, we can experience translational movement without RDW modulation. Experimental results show that the smaller the size of the Guardian space, the greater the amplification of translational movement by the RDW, and thus the larger the error due to small translational movements.

\subsection{Mapping}

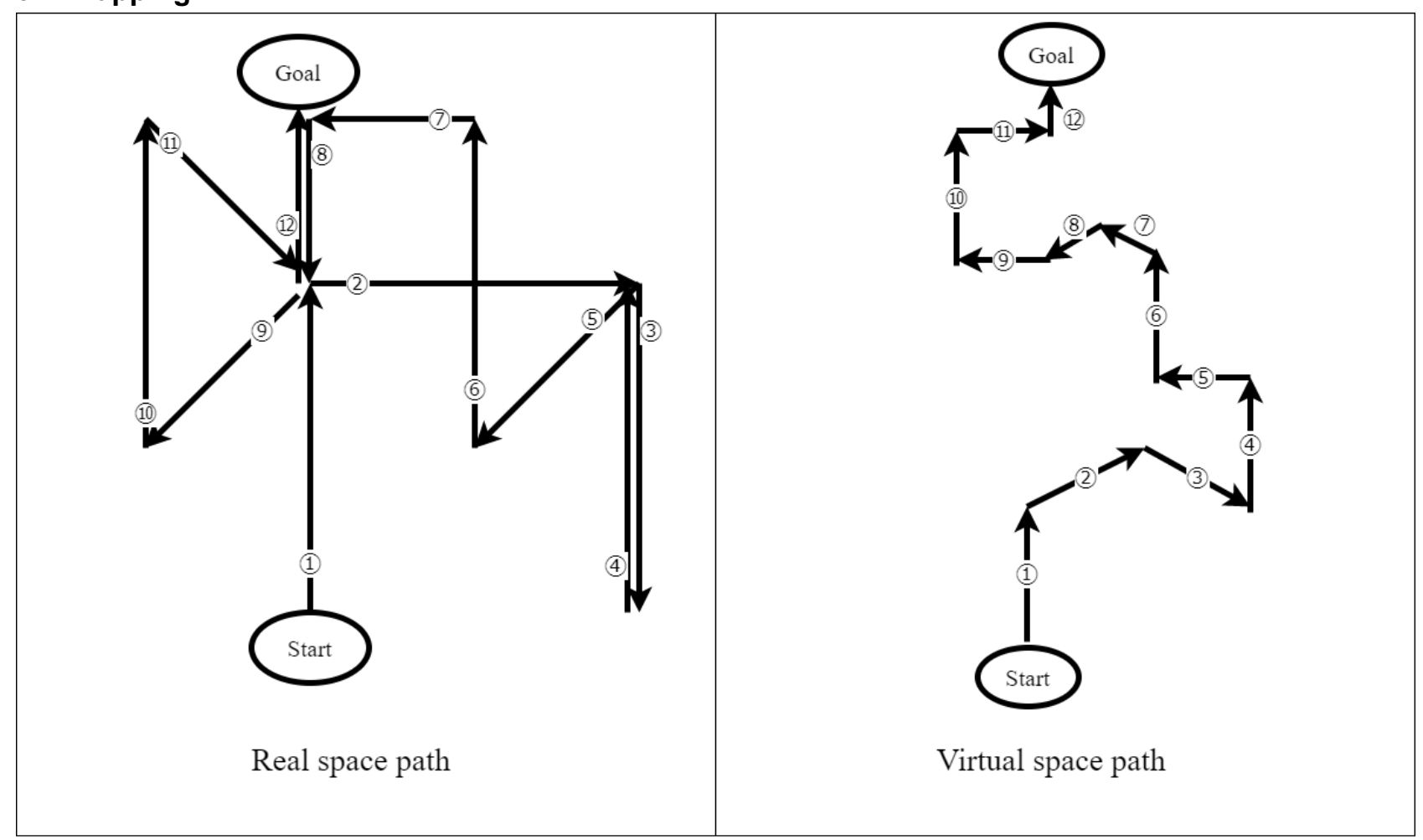

Fig. 2. Juxtaposition of real and virtual paths 


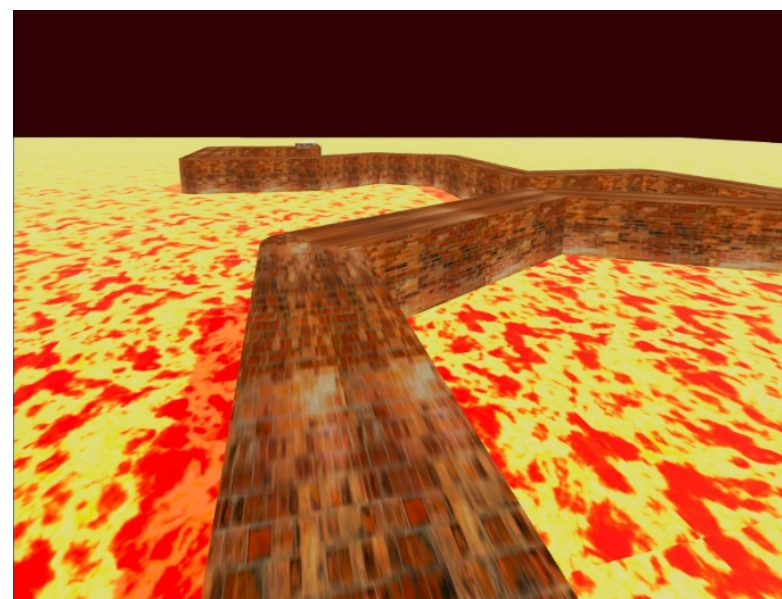

Fig. 3. First-person (egocentric) view, showing virtual path and surrounding molten lava

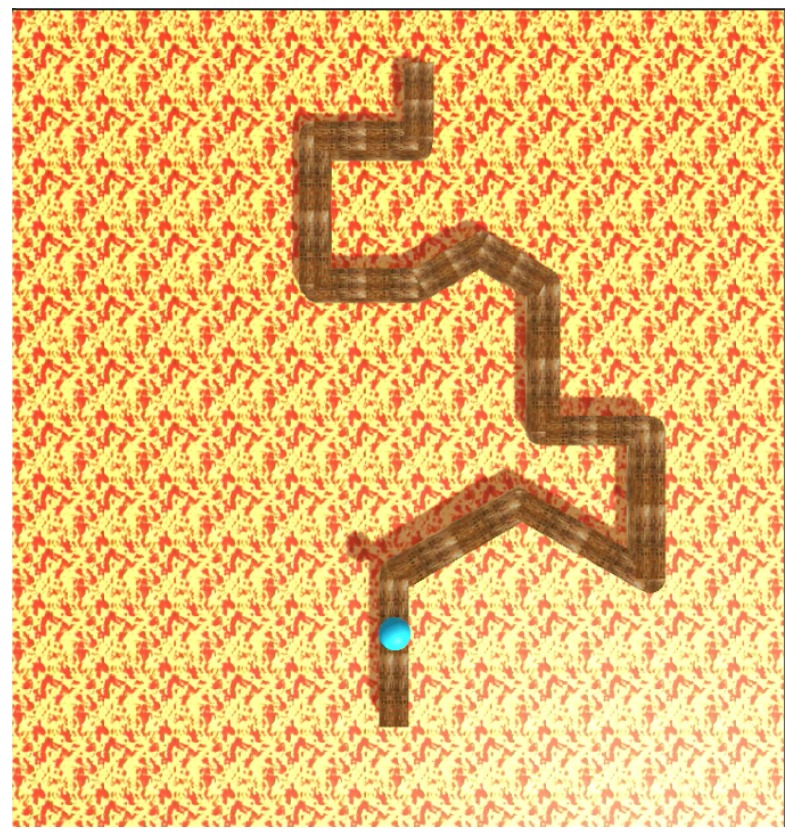

Fig. 4. Third-person (exocentric) view

\section{Conclusion and Future Work}

As mentioned in the previous section, the smaller the real space, the more minute movements are extended in the virtual space, leading to the user unexpectedly leaving the path and consequently out of Guardian Space. This is because the magnitude of the translation gain is inversely proportional to the size of the Guardian Space since movement is amplified according to size of the path in the virtual space. In addition, this method can only simulate a predetermined path, and one cannot truly roam in virtual space. It is effective for content that follows a predetermined path, such as mazes and trails, but it is not suitable for content that allows players to freely explore a large space, such as in contemporary FPS games.

The aforementioned problems could be addressed by experimenting with the possibility of changing the amount of dynamic manipulation by adjusting each gain variable according to the user's position in the Guardian Space.

\section{References}

1. H. Iwata. (2000). Locomotion interface : Locomotion Interface Using Omni-directional Infinite Surface. Trans. of the Virtual Reality Society of Japan, 5(2), 853-862.

2. H. Iwata, \& F. Nakaizumi. (2000). The GaitMaster: Locomotion Interface for Uneven Virtual Surface. Trans. of the Virtual Reality Society of Japan, 5(2), 863-866.

3. Virtuix Omni https://www.virtuix.com

4. CyberShoes https://www.cybershoes.io

5. K. Matsumoto, T. Narumi, Y. Ban, T. Tanikawa, \& M. Hirose. (2018). Curvature Manipulation Type Redirected Walking using Visuo-haptic Interaction, Trans. Virtual Reality Society of Japan, 23(3), 129-138.

6. Y. Saito, Y. Murosaki, R. Ono, \& H. Hada. (2018). Redirected Walking Method Based on Perception Characteristics of Wind Direction, IPSJ SIG Technical Reports, 2018(7), 1-5.

7. K. Satoh, R. Tadoh, \& S. Serikawa. (2019). Proposal of Curvature Manipulation Type of Guidance Method by Moving Sound Source for Virtual Reality, Proc. Institute of Industrial Applications Engineers Annual Conf., 2019 (pp. 7-8).

8. C. Nakagaito, Y. Hashimoto, M. Oka, \& K. Kato. (2017). Redirected walking by attention manipulation using VR and foot sole haptic. Proc. Annual Conf. of Japanese Society for Artificial Intelligence. (pp. 1G33-1G33).

9. F. Steinicke, G. Bruder, L. Kohli, J. Jerald, \& K. Hinrichs. (2008). Taxonomy and implementation of redirection techniques for ubiquitous passive haptic feedback. Proc. Int. Conf. on Cyberworlds (pp. 217-223).

10. Oculus Quest

https://www.oculus.com/quest-2/

11. Unity https://unity.com

12. Photon https://www.photonengine.com 